\title{
A Remedy for Nonstationarity in Background Transition Regions for Real Time Hyperspectral Detection ${ }^{2}$
}

\author{
A. Schaum \\ Naval Research Laboratory \\ 4555 Overlook Ave \\ Washington D.C. 20375 \\ 202 767-9366 \\ schaum@nrl.navy.mil
}

\begin{abstract}
Real-time hyperspectral systems rely on a variety of statistical detection methods that all require the adaptive estimation of first- and second-order background statistics. Inadequate adaptation can occur when an airborne sensor encounters a physical transition area (e.g. forest to meadow), resulting both in excessive false detections, and in the failure to detect targets that have been strategically positioned in boundary areas. Here we describe a two-component statistical mixture model that gracefully grows the capabilities of deployed systems to adapt to abrupt transitions. First, the parameters of a bi-modal Gaussian model are found with a modified Expectation Maximization technique. Next, the detection phase of processing tests for hypotheses that allow mixtures of these background components, with unknown mixing fraction estimated as part of a generalized likelihood ratio test (GLRT). A straightforward application of existing real time spectral whitening modules allows the simultaneous diagonalization of the estimated covariance matrices of both primary mixture components. This generates, for both anomaly- and signature-based detection, only a small additional computational burden, consisting of a standard Newton-Raphson algorithm applied to a polynomial function.
\end{abstract}

\section{TABLE OF CONTENTS}

1. INTRODUCTION ............................................................ 1

2. REAL TIME HYPERSPECTRAL DETECTION ........... 1

3. DeteCtion With UNKNOWN PARAMETERS ........ 4

4. A BACKGROUND MODEL FOR TRANSITION

AREAS: THE TWO-COMPONENT SMM.

5. SUMMARY 6

REFERENCES

BIOGRAPHY ................................................................ 9

\section{INTRODUCTION}

The Naval Research Laboratory (NRL) maintains a suite of target detection algorithms designed to operate on the out-

\footnotetext{
${ }^{1}$ U.S. Government work not protected by U.S. copyright.

2 IEEEAC paper \#1316, Version 6, Updated October 24, 2005.
}

puts of its various hyperspectral sensor systems. These include VNIR (visible/near infrared: $0.4 \rightarrow 1.0 \mu \mathrm{m}$ ), SWIR (short wave: $1.0 \rightarrow 2.5 \mu \mathrm{m}$ ) and in the near future LWIR (long wave infrared: $8.0 \rightarrow 12.0 \mu \mathrm{m}$ ). The core detection algorithms are nearly independent of the wavelength sensing region.

The Civil Air Patrol (CAP) is also in the process of deploying up to 16 operational VNIR systems (called "ARCHER" [1]) based on these algorithms, which were developed by NRL in collaboration with Space Computer Corporation (Los Angeles, CA).

The standard detection algorithms employed in all these systems achieve low false alarm rates over regions where quasi-stationarity of background statistics is a good approximation. However, this condition fails famously over background transition regions, where false alarms tend to concentrate. This paper proposes a solution that leverages the existing signal processing modules to implement a simple version of a probabilistic extension of linear mixing models called the SMM (Stochastic Mixing Model [2]).

\section{ReAL Time HyPERSPECTRAL DETECTION}

In the airborne sensor systems described above, the onboard signal processor accepts blocks of hyperspectral data that are used to serially update estimates of radiance statistics of the physical background (usually situated beneath an airborne collection platform). These statistics are required for constructing all the onboard real time detection algorithms. Initial estimates

$$
\begin{aligned}
& \mu=\frac{1}{N} \sum_{i=1}^{N} x_{i} \\
& C=\frac{1}{N} \sum_{i=1}^{N}\left(x_{i}-\mu\right)\left(x_{i}-\mu\right)^{\dagger}
\end{aligned}
$$


of the mean and covariance matrix describing the background distribution of the data are constructed from a block of digitized background vectors $x_{i}$, representing the sensorderived estimate of spectral radiance at pixel $i$. The matrix transpose operation is denoted by $\dagger$.

Each component of $x_{i}$ in this vector notation corresponds to a different discretized wavelength. The dimension is typically at least several dozen, although the final value usually results from a local spectral integration of the several hundred bands collect by a hyperspectral sensor.

The approximations in Equation (1) are the maximum likelihood estimates of the first- and second-order statistics of a Gaussian model of the background probability density function (pdf)

$$
p_{B}(x)=(\sqrt{2 \pi})^{-N}\|C\|^{-\frac{1}{2}} \exp \left(-\frac{1}{2}(x-\mu)^{\dagger} C^{-1}(x-\mu)\right)
$$

with $\|C\|$ the determinant of $C$.

Another model that has been implemented in real time, but which is lately de-activated in field tests, allows a multimodal generalization of the form in (2). Gaussian spectral clustering (GSC) uses a modified version of the standard Expectation Maximization algorithm [3] to estimate a preset number $N_{C}$ of distinct mean values and associated covariance matrices. Selection of the number $N_{C}$ of clusters has never been automated, because algorithms based on this background model are not viewed as reliable improvements over unimodal ones. However, the existence of a real time version of GSC proves useful in the detection models proposed below.

After the initialization phase is completed and as the sensor ingests more data, the background statistics are updated adaptively, and the old statistics are weighted with a "forgetting factor" so that they can be gradually deemphasized. The implicit assumption of this technique is quasistationarity of the background spatial distribution of spectra.

Using these statistical estimates, two algorithms are currently implemented, called "RX" and "LMF." These are prototype representatives of two general classes of algorithm: anomaly detection and signature-based detection. We will refer to RX and LMF as the "standard" algorithms. In Section (3) we introduce newer concepts aimed at solving a commonly encountered type of nonstationarity.

\section{The RX Anomaly Detector}

The standard (RX) anomaly detector is a test for rare pixels, as determined by the model pdf in Equation (2). Unusual pixel values - corresponding to a pdf value less than some threshold value - are labeled targets. This process is logically equivalent to a test of the form

$$
(x-\mu)^{\dagger} C^{-1}(x-\mu)>k \equiv \text { threshold }
$$

for deciding that a pixel with radiance vector $x$ contains a target. The values of $\mu$ and $C$ are defined in Equation (1); no distinction is made between their estimated and "true" values. The spectral region corresponding to a target declaration is the exterior of a hyperellipsoid (Figure 1), defined by Equation (3).

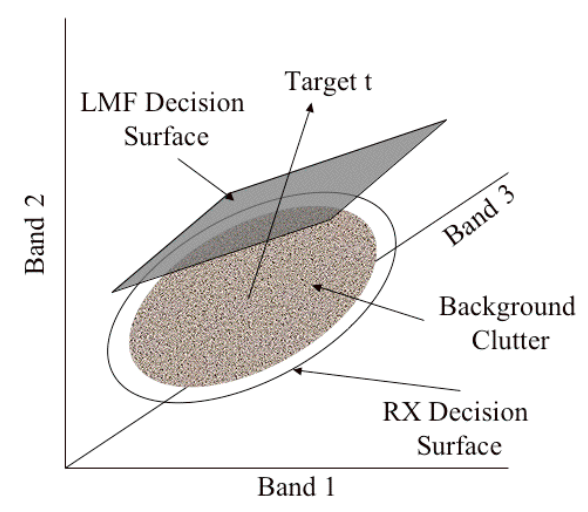

Figure 1. Gaussian clutter model with decision surfaces for anomaly detection and linear matched filter.

\section{The Linear Matched Filter}

The standard signature-based detector is the linear matched filter (LMF). A mean target signature $t$ is assumed to be available for use in a detection algorithm. Knowing an approximate value of the target signature allows the construction of a more selective decision surface than defined by Equation (3).

For the LMF, a target is declared at any pixel $x$ satisfying

$$
(t-\mu)^{\dagger} C^{-1}(x-\mu)>k
$$

The corresponding decision surface is a hyperplane perpendicular to the vector $C^{-1}(t-\mu)$. To see why this choice of hyperplane is a good one, it is convenient to view the data in a transformed representation of radiance space.

\section{Whitened Formulation}

The RX and LMF detectors admit simple geometrical interpretations. In spectral space their decision surfaces account for the non-sphericity of the background distribution. The RX decision boundaries (Figure 1) are nothing but the surfaces of constant background pdf (see Equation (2)). And instead of using the hyperplane orthogonal to the line between background and target means, $(t-\mu)$, the LMF decision surface is rotated (by the action of the matrix $C^{-1}$ ) to account for a possible asymmetry in the shape of the background distribution. 
An intuitive explanation of these algorithms is easier if the asymmetry is removed. This can be accomplished by a "whitening" transformation of the radiance variable $x$

$$
w \equiv W(x-\mu)
$$

The matrix $W$ is defined by

$$
W=C^{-\frac{1}{2}} \equiv \Lambda D^{-\frac{1}{2}} \Lambda^{\dagger}
$$

where

$$
C \equiv \Lambda D \Lambda^{\dagger}
$$

is a singular value decomposition of the matrix $C, \Lambda$ is an orthonormal matrix (of eigenvectors), $\Lambda^{\dagger}$ is its adjoint, and $D$ is a non-negative diagonal matrix of eigenvalues. The zero-mean whitened variable $w$ replaces the radiance variable $x$, and the particular forms of RX and LMF algorithms become obvious choices for detection algorithms.

The RX decision (Equation (3)) is equivalent to:

$$
w^{2}=w \cdot w>k
$$

that is, the Euclidean length of the vector $w$ is compared to a threshold.

In whitened variables, the LMF statistic (Equation (4)) becomes a Euclidean inner product

$$
w_{t} \bullet w>k
$$

where the whitened target signature is

$$
w_{t} \equiv W(t-\mu)
$$

These simplifications occur because the variable $w$ is zeromean and has a covariance matrix equal to the identity:

$$
\frac{1}{N} \sum_{i=1}^{N}\left(w_{i}\right)\left(w_{i}\right)^{\dagger}=I \text {. }
$$

When viewed in terms of the variable $w$, Figure 1 is transformed into Figure 2, the whitened space in which the background distribution appears more spherically symmetric. (The variance is the same in all directions.) According to Equation (8), anomalies are simply any pixels situated sufficiently far from the mean of the background distribution.

Because $W$ is a linear transformation, it maps the hyperplane of Figure 1 into another hyperplane in Figure 2. But in the whitened space, the vector $w_{t}$ defines the only preferred direction, to which the hyperplane decision surface must therefore be orthogonal. Equation (9) is the algebraic expression of this geometrical interpretation of the LMF decision surface. The test for a target consists of measuring a pixel's distance from the hyperplane.

\section{Covariance Equalization}

The dot product forms of RX (Equation (8)) and LMF (Equation (9)) in whitened space make the final stage of detection signal processing a straightforward calculation of a few sums of products. However, the more important motivation for decomposing the detection processing to make whitening the initial step stemmed from studies of how background statistics of a given geographic area vary with time.

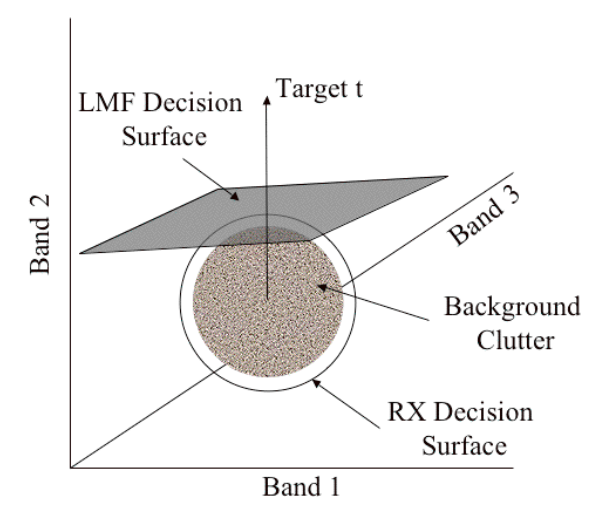

Figure 2. Simplified geometry in whitened variables. The coordinate origin is actually at the center of the sphere.

It has been demonstrated on hyperspectral visible and infrared data sets that one can often accurately estimate the spectral shape of a pixel $x$ (in the open) at one time from the corresponding value $x^{\prime}$ at another time (as much earlier as several weeks) with the approximation

$$
\hat{x}=\mu+W^{-1} W^{\prime}\left(x^{\prime}-\mu^{\prime}\right) \approx x
$$

where $\mu^{\prime}, W^{\prime}$ are defined as in Equation (1), but are derived from measurements made at the earlier time. This method of estimation is called Covariance Equalization (CE), because the second-order (as well as first-order) statistics of the approximation $\hat{x}$ are guaranteed to reproduce those of $x$ exactly.

Guided by Equation (5), we define the whitened estimate as

$$
\hat{w}=W(\hat{x}-\mu)
$$

so that from Equation (12)

$$
\hat{w}=w^{\prime} \approx w,
$$


with $w^{\prime}$ defined as the earlier-time version of Equation (5).

Therefore, to the extent that CE acts as a good estimator of spectral radiance, the whitened pixel values $w^{\prime}$ from an earlier time are good estimates of the corresponding ones $w$ at a later time. Whitened hyperspectral imagery forms an invariant representation of the background, at least in terms of first and second moments.

This principle has been invoked to achieve extremely good change detection performance. For real time implementation, the adaptive background statistical estimates are used to generate locally whitened pixels, which are compared to a similarly processed data set of pixels from an earlier mission. One can expect the detection statistic $w-\hat{w}$ to remove most of the background clutter, except at pixels whose radiance have been changed anomalously, as by the insertion of a new object, or by an environmental event. The RX algorithm can be applied to $w-\hat{w}$ to convert this vector error signal into a scalar detection statistic.

CE is also used to estimate target signatures as they appear in a new environment, using their measured values in an old one. For a target, an equation analogous to (12)

$$
\hat{t}=\mu+W^{-1} W^{\prime}\left(t^{\prime}-\mu^{\prime}\right) \approx t
$$

predicts a target signature. The analogue of (14) is then

$$
\hat{w}_{t}=w_{t}^{\prime} \approx w_{t} \text {. }
$$

Therefore, if the required target signature $t$ is not available for use in the LMF, but an earlier version $t^{\prime}$ is known, a matched filter based on an estimated value of $t$ can still be implemented. Combining Equations (16) and (9) yields the simple form of the matched filter based on an earlier signature:

$$
w_{t}^{\prime} \bullet w>k
$$

Just as only adaptively whitened background pixels $w^{\prime}$ need to be stored for the change detection application, so too only the whitened form of target signature $w_{t}^{\prime}$, as defined by an earlier mission, needs to be stored to implement the LMF in a search for a current instance of the target.

\section{Detection With UnKNoWn Parameters}

Both RX and LMF detectors are based on a simple statistical model of hyperspectral clutter. And the adaptive estimation of the requisite background statistics allows for gradual changes in the parameters of the model as a mission progresses. But it cannot account for the abrupt change in sensed radiance that commonly occurs when fields give way to forests, or for any other discontinuous change in back- ground composition. Figure 3 illustrates how the presence of two distinct background constituents (spectral clusters) can confuse a Gaussian model designed for a unimodal distribution. Because it fails to account for distinct clusters, the Gaussian shape does not fit the data well. The covariance matrix computed by both RX and LMF is corrupted by an important deviation from the model, which causes a separation of cluster means to be mistaken for variability within a unimodal distribution. Accurate detection can then require artificially large separations of a target from the clutter mass.

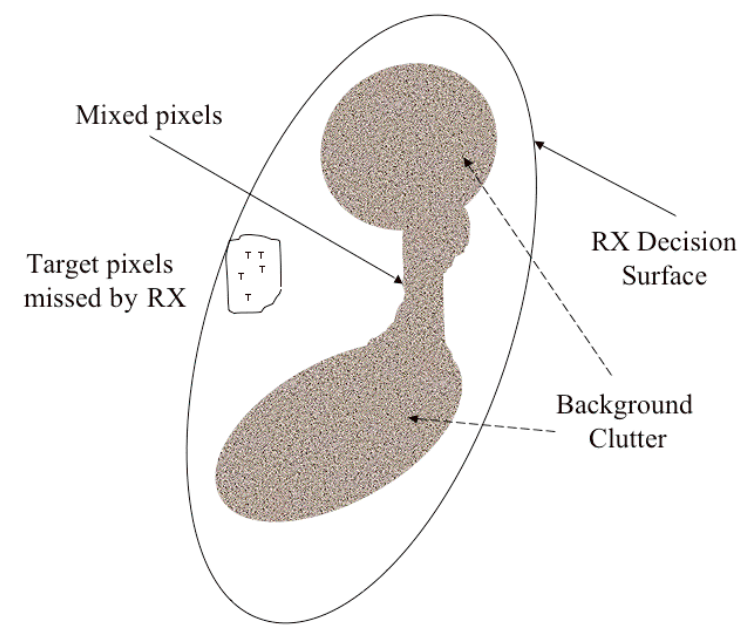

Figure 3. The RX ellipsoidal decision surface becomes artificially large when clusters of background constituents are not recognized as distinct.

The GSC model allows for multi-modal backgrounds, but it assumes that every sensed pixel belongs to one cluster. Mixed pixels, which typify transition regions between terrain types, are likely false alarms (Figure 4) in a GSC-based target detection scheme.

\section{Likelihood Ratio Tests}

A reliable background model can aid in anomaly detection, by motivating a test to assess the likelihood that a pixel is consistent with it. One typically constructs a metric to rate the degree to which a test pixel deviates from pixels allowed by the model. For signature-based methods, a related common practice [4,5] consists of deleting any background component of a test pixel and then comparing the residual to a target model (subjected to the same deletion).

A more systematic approach invokes an approximation to the optimal detection algorithm, which is known to be a likelihood ratio (LR) test.

The performance of an algorithm designed to distinguish between two alternatives ( $x$ is from the background, or from a target) can be characterized by two parameters, commonly chosen as the target detection probability, $P_{D}$, and the false alarm probability $P_{F}$. And a fundamental result (attributed in [6] to Birdsall) from binary decision 
theory states that for any cost function that depends on only these two variables, the optimal (minimum average cost) algorithm for correctly classifying a target with value $x$ is always of the general form

$$
\frac{p_{T}(x)}{p_{B}(x)}>k,
$$

where the threshold $k$ depends on the particulars of the cost model. Equation (18) is also the form of test appropriate for meeting the "Neymann-Pearson" (N-P) criterion, which requires maximization of $P_{D}$ for any pre-assigned value of $P_{F}$. (Not surprisingly, this criterion can be cast as a cost minimization problem.) N-P is a common design goal in wide-area autonomous detection applications.

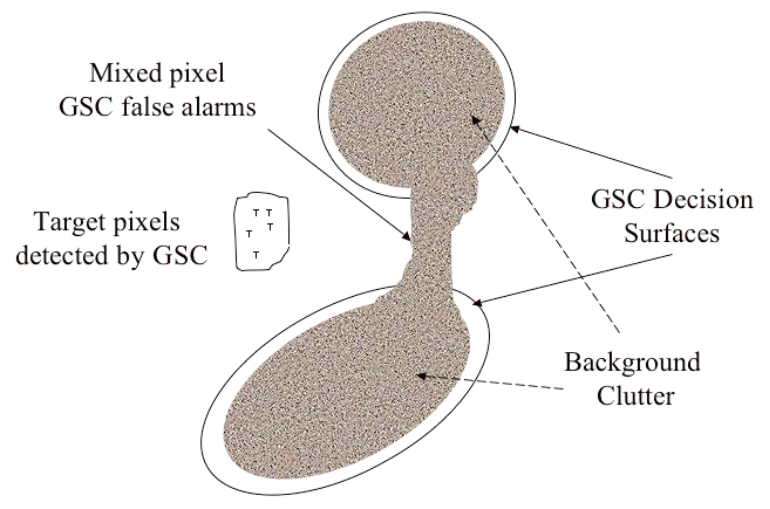

Figure 4. Gaussian spectral clustering can recognize distinct background constituents and better distinguish target from background, but it does not accommodate mixed pixels.

Equation (18) is useful, of course, only if good estimates of the target and background pdfs are available. In the more common situation, one relies on parametric models of the background and target, and then uses the sensed data, the pixel value $x$, to estimate the values of the parameters. (Used in combination with Equation (18), this is called a GLR (generalized likelihood ratio) test.) When applied to the binary decision problem, a fundamental dilemma appears: One doesn't know which of the two distributions $x$ was drawn from.

This is of little concern when estimating bulk background statistics, as in Equation (1) for a one-cluster Gaussian model. Contamination by a few target pixels appearing among the thousands of terms in the sums has minimal effect on the estimates (see however, [7], for some surprising, if unexplained results). And we are interested here in situations for which the number of background pixels far exceeds the number of target pixels.
The story is much different, however, for estimating target parameters, reliable values of which are harder to come by. These require a modified technique.

The standard approach to this problem of unknown parameters replaces the LR test with a GLR test:

$$
\frac{\operatorname{Max}_{\left\{t_{i}\right\}} p_{T}\left(x:\left\{t_{i}\right\}\right)}{\operatorname{Max}_{\left\{b_{i}\right\}} p_{B}\left(x:\left\{b_{i}\right\}\right)}>k,
$$

where $\left\{t_{i}\right\}\left(\left\{b_{i}\right\}\right)$ are the unknown but deterministic parameters associated with the target (background) model.

This paper considers several models for the distribution of background pixels $x_{B}$. But it in each case, the relationship of target to background is the same. Pixels $x_{T}$ that contain targets are always treated as random variables that obey the "additive target" model, according to which

$$
x_{T}=t+x_{B} .
$$

This is certainly well-motivated for extreme sub-pixel targets, for which a measurement $x_{T}$ of a pixel with target must include most of a background pixel as well. But it is also reasonable for larger targets that can interact with the local background (e.g. through reflections) or when a target pixel is corrupted by "adjacency effects" (atmospheric scattering of neighboring background pixels into the geometrical field of view of a target pixel), which are important for long-range stand-off sensing.

Because here we always treat $t$ as deterministic-although perhaps unknown-Equation (20) implies that the target and background pdfs are related:

$$
p_{T}\left(x:\left\{t_{i}\right\}\right)=p_{B}\left(x-t:\left\{b_{i}\right\}\right)
$$

As segue to the proposed SMM-based algorithm, which relies on a GLR test, we note that both RX and the LMF can also be derived from GLR tests, if the Gaussian background model is assumed. In particular, when Equation (1) defines all the parameters of the background distribution in Equation (2), no free parameters need to be estimated on a per/pixel basis as part of the denominator in Equation (19). And if $t$, the target/background mean signature difference, is the only target parameter, then Equation (21) reduces to

$$
p_{T}(x)=p_{B}(x-t) .
$$

If, for example, we assume that the vector $t$ is completely unknown then, from Equations (2) and (22), the numerator of Equation (19) is maximized by the choice $t=x$ and is, therefore, independent of $x$. Then the GLR test reduces to 
the comparison of the background pdf to a threshold or, equivalently, to Equation (3), the RX test.

In the case where only the direction of $t$ (relative to the background mean) is known, the numerator of Equation (21) does depend on the value of $x$. An instructive if tedious argument (easily made in the whitened space) shows that Equation (19) then leads to the linear matched filter. The LMF is usually thought of as the optimal hypothesis test for Gaussian distributions, in which $t$ is treated as fully known (in which case the GLR reduces to the LR). It actually solves a wider class of problems.

\section{A BACKGROUND MODEL FOR TRANSITION Areas: The Two-CoMponent SMM}

Pixels from boundary areas can consist of arbitrary mixtures of materials that appear in purer form in (geographically) adjacent areas. While their number might account for only a small fraction of a training set, these transition area pixels can be the most significant source of false alarms degrading performance in a high performance autonomous system. Wide-area remote sensing systems often have miniscule error rate requirements.

In spectral space (Figure 4) the mixed pixels appear between the clusters representing the purer materials. As discussed above, neither the unimodal Gaussian nor the GSC models account for such mixed signals. Here we propose a background model designed to accommodate this transition area problem. The general model appeared in [8], but variations of it have appeared for target detection [9] and for other purposes [10]. Here we allow for two pure classes of background material, each of whose distribution is modeled as Gaussian, but with separate mean values $\mu_{1}, \mu_{2}$ and covariance matrices $C_{1}, C_{2}$. Furthermore, mixed pixels are now allowed

$$
x=f x_{1}+(1-f) x_{2} \quad(0 \leq f \leq 1),
$$

$x_{\mathrm{i}}$ being drawn from class $i$. The mixtures are treated mathematically as sums of weighted independent Gaussian random variables, whose radiance distributions are therefore also Gaussian, with parameters

$$
\begin{aligned}
& \mu=f \mu_{1}+(1-f) \mu_{2} \\
& C=f^{2} C_{1}+(1-f)^{2} C_{2} .
\end{aligned}
$$

This form for the mean is implied by (23); the particular form for $C$ assumes that the spectral/spatial correlation length $l$ is greater than one. ${ }^{3}$ For the real time hyperspectral sensing systems described above, the two sets of statistics can be estimated with the extant GSC signal processing

\footnotetext{
${ }^{3}$ As $l \rightarrow 0$ a more appropriate model for $C$ is: $C=f C_{1}+(1-f) C_{2}$.
}

module, with $N_{c}=2$. However, this constraint on the number of clusters allows the derivation of a nearly closed form solution to the GLR test statistic in Equation (19).

In the detection decision, every test pixel is assumed to be drawn from a mixture distribution, with a pdf that is again described by the form in Equation (2). Now, however, the parameters $\mu$ and $C$ depend, through Equation (24), on the unknown parameter $f$. Its value must be determined implicitly at each pixel from the maximum likelihood technique and then used in Equation (19) in a GLR test.

\section{Simultaneous Diagonalization of Covariance Matrices}

To simplify maximization of the denominator, we again exploit an existing software module, which implements the spectral whitening function. Whitening with a covariance matrix $C$ involves first expressing it as an SVD (Equation (7)), then computing the inverse square roots of the diagonal part $(D)$, as in Equation (6).

The whitening module can be exploited to diagonalize both $C_{1}$ and $C_{2}$ simultaneously with little extra computational burden. The process has a simple geometrical interpretation.

In Figure 5 the two symmetric covariance matrices $C_{1}$ and $C_{2}$ are represented as ellipsoids (the x-representation). This is possible because any symmetric matrix admits a singular value decomposition of the form in Equation (6). The columns of $\Lambda$ are the eigenvectors, and correspond to the principal axes of the ellipsoid. The eigenvalues along the diagonal of $D$ define the (squared) lengths of these axes.
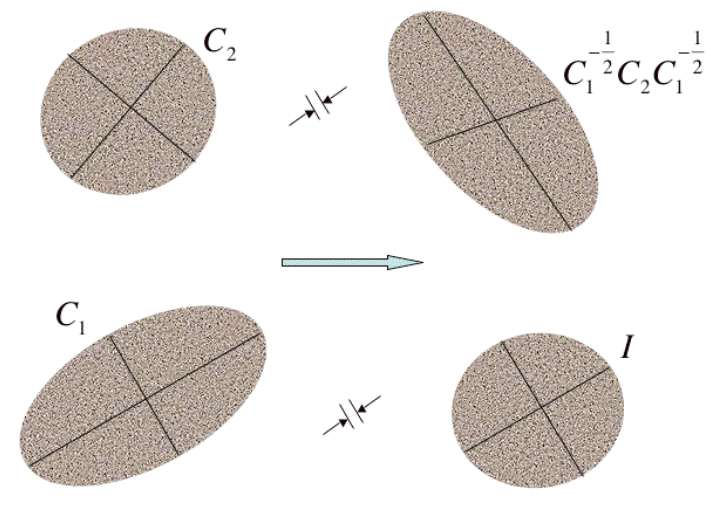

X-representation

y-representation

Figure 5. A whitening transformation based on covariance matrix 1 compresses the $x$-representation of cluster 1 into a spherically symmetric y-representation and distorts cluster 2 .

To accomplish the diagonalization, first we whiten with $C_{1}$. That is, we express all data in the variable

$$
y=C_{1}^{-\frac{1}{2}}(x-\mu)
$$


(The offset $\mu$ is included for later convenience.) Cluster 1 has mean value (denoted by $<>$ )

$$
\left\langle y_{1}\right\rangle=\left\langle C_{1}^{-\frac{1}{2}}\left(x_{1}-\mu\right)\right\rangle=C_{1}^{-\frac{1}{2}}\left(\mu_{1}-\mu\right),
$$

and so, in the $y$-variable, the covariance matrix of cluster 1 becomes, after some simplification

$$
\begin{aligned}
& \left\langle\left[y_{1}-\left(C_{1}^{-\frac{1}{2}}\left(\mu_{1}-\mu\right)\right)\right]\left[y_{1}-\left(C_{1}^{-\frac{1}{2}}\left(\mu_{1}-\mu\right)\right)\right]^{\dagger}\right\rangle \\
& =C_{1}^{-\frac{1}{2}}\left\langle\left(x_{1}-\mu_{1}\right)\left(x_{1}-\mu_{1}\right)^{\dagger}\right\rangle C_{1}^{-\frac{1}{2}}=C_{1}^{-\frac{1}{2}} C_{1} C_{1}^{-\frac{1}{2}}=I .
\end{aligned}
$$

For cluster 2, a similar calculation produces the covariance matrix $C_{1}^{-\frac{1}{2}} C_{2} C_{1}^{-\frac{1}{2}}$. Generally, the transformation in Equation (25) induces the covariance matrix changes:

$$
C_{i} \rightarrow C_{1}^{-\frac{1}{2}} C_{i} C_{1}^{-\frac{1}{2}} \quad(i=1,2)
$$

in the y-representation. As Figure 5 indicates, cluster 1 is compressed in its eigenvector directions by the whitening transformation $C_{1}^{-\frac{1}{2}}$ until it is spherically symmetric, with covariance equal to the identity matrix $I$. Cluster 2 is distorted by the same whitening operation.

The advantage lies in the fact that the transformed covariance matrices now commute ( $I$ commutes with anything).

This means that the y-representations of the covariance matrices can, in turn, be simultaneously diagonalized by an orthonormal transformation, in particular, by a rotation. Let the SVD of the transformed second covariance matrix be

$$
C_{1}^{-\frac{1}{2}} C_{2} C_{1}^{-\frac{1}{2}} \equiv \Lambda D \Lambda^{\dagger}
$$

with $\mathrm{D}$ a diagonal and $\Lambda$ an orthonormal matrix. Then form the rotated variable

$$
z=\Lambda^{\dagger} y
$$

The covariance matrices in $z$-space are found by another similarity transformation (Figure 6). This time, instead of (28), the similarity transformations are governed by $\Lambda$ :

$$
\begin{aligned}
I & \rightarrow \Lambda^{\dagger} I \Lambda=I \\
C_{1}^{-\frac{1}{2}} C_{2} C_{1}^{-\frac{1}{2}} & \rightarrow \Lambda^{\dagger}\left(\Lambda D \Lambda^{\dagger}\right) \Lambda=D
\end{aligned}
$$

where (29) has been used. Therefore, in $z$-space, both matrices are diagonal. This greatly simplifies the optimization calculation.

To summarize, in the variable

$$
z=\Lambda^{\dagger} C_{1}^{-\frac{1}{2}}(x-\mu)
$$

pixels in cluster 1 have covariance matrix

$$
\left\langle\left(z_{1}-\mu_{z_{1}}\right)\left(z_{1}-\mu_{z_{1}}\right)^{\dagger}\right\rangle=I
$$

and pixels in cluster 2 have covariance matrix

$$
\left\langle\left(z_{2}-\mu_{z_{2}}\right)\left(z_{2}-\mu_{z_{2}}\right)^{\dagger}\right\rangle=D
$$

which is diagonal and derives from the SVD in Equation (29). These results are now used in the maximum likelihood calculations needed for evaluating Equation (19).

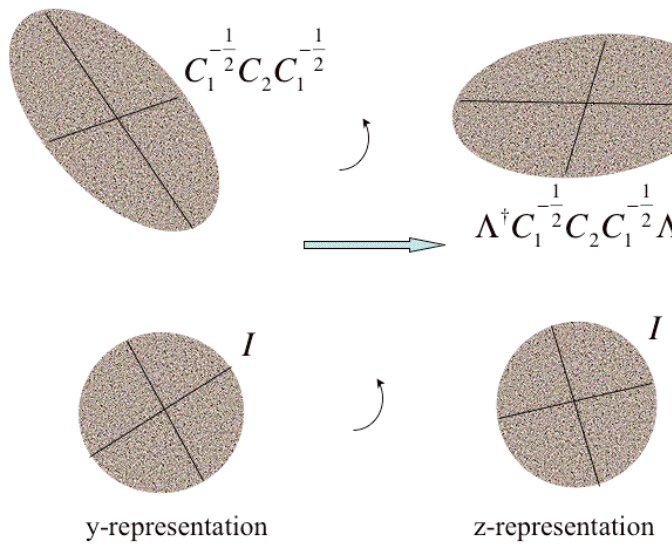

Figure 6. A rotation derived from the decomposition in Equation (29) completes the simultaneous diagonalization of the covariance matrices. The eigenvectors of cluster 2 are caused to align with the Cartesian axes (top), which can also serve as eigenvectors for the spherically symmetric cluster 1 (bottom).

\section{Simplified Maximum Likelihood Calculation}

Maximizing the denominator of Equation (19) is equivalent to minimizing its negative logarithm, and hence, from Equation (2), to minimizing

$$
L(f) \equiv \frac{1}{2} \ln (\|C\|)+\frac{1}{2}(x-\mu)^{\dagger} C^{-1}(x-\mu),
$$

with \|\| denoting a determinant. The $f$ dependence is described by Equation (24), which also yields 


$$
\begin{aligned}
C & =f^{2} C_{1}+(1-f)^{2} C_{2} \\
& =C_{1}^{\frac{1}{2}}\left[f^{2}+(1-f)^{2} C_{1}^{-\frac{1}{2}} C_{2} C_{1}^{-\frac{1}{2}}\right] C_{1}^{\frac{1}{2}} .
\end{aligned}
$$

Because square matrices can be commuted without changing the determinant of their product, and with the help of Equation (29),

$$
\begin{aligned}
\ln \|C\| & =\ln \left\|\left[C_{1}\right]\left[f^{2}+(1-f)^{2} \Lambda D \Lambda^{\dagger}\right]\right\| \\
& =\ln \left\|C_{1}\right\|+\ln \left\|\Lambda\left[f^{2}+(1-f)^{2} D\right] \Lambda^{\dagger}\right\| \\
& =\ln \left\|C_{1}\right\|+\ln \left\|\left[f^{2}+(1-f)^{2} D\right]\right\| \\
& =\ln \left\|C_{1}\right\|+\sum_{i} \ln \left(\left[f^{2}+(1-f)^{2} D_{i i}\right]\right) .
\end{aligned}
$$

After dropping the first term, which has no $f$ dependence, the task becomes minimizing

$$
\begin{aligned}
& L(f) \rightarrow \\
& \frac{1}{2} \sum_{i} \ln \left(\left[f^{2}+(1-f)^{2} D_{i i}\right]\right)+\frac{1}{2}(x-\mu)^{\dagger} C^{-1}(x-\mu) .
\end{aligned}
$$

From Equations (32) and (29), the second term can be written

$$
\begin{aligned}
& \frac{1}{2} z^{\dagger} \Lambda^{\dagger} C_{1}^{\frac{1}{2}}\left[f^{2} C_{1}+(1-f)^{2} C_{2}\right]^{-1} C_{1}^{\frac{1}{2}} \Lambda z \\
& =\frac{1}{2} z^{\dagger}\left[f^{2}+(1-f)^{2} \Lambda^{\dagger} C_{1}^{-\frac{1}{2}} C_{2} C_{1}^{-\frac{1}{2}} \Lambda\right]^{-1} z \\
& =\frac{1}{2} z^{\dagger}\left[f^{2}+(1-f)^{2} D\right]^{-1} z .
\end{aligned}
$$

Therefore, $L(f)$ from (38) becomes

$$
\frac{1}{2} \sum_{i}\left\{\ln \left[f^{2}+(1-f)^{2} D_{i i}\right]+z_{i}^{2}\left[f^{2}+(1-f)^{2} D_{i i}\right]^{-1}\right\} .
$$

Note that $z$ depends on $f$ (Equation (32)).

$$
\begin{aligned}
z & =\Lambda^{\dagger} C_{1}^{-\frac{1}{2}}\left\{x-\left[f \mu_{1}+(1-f) \mu_{2}\right]\right\} \\
& \equiv u+f w \quad \text { with } \\
u & \equiv \Lambda^{\dagger} C_{1}^{-\frac{1}{2}}\left(x-\mu_{2}\right) \\
w & \equiv \Lambda^{\dagger} C_{1}^{-\frac{1}{2}}\left(\mu_{2}-\mu_{1}\right) .
\end{aligned}
$$

Using (41) in (40) and extremizing with standard calculus yields an equation for $f$ :

$$
\begin{aligned}
0 & =\sum_{i} \frac{f-(1-f) D_{i i}+\left(u_{i}+f w_{i}\right) w_{i}}{f^{2}+(1-f)^{2} D_{i i}} \\
& -\sum_{i} \frac{\left[u_{i}+f w_{i}\right]^{2}\left[f-(1-f) D_{i i}\right]}{\left[f^{2}+(1-f)^{2} D_{i i}\right]^{2}} .
\end{aligned}
$$

One can rationalize this equation and thereby show that solving (42) is equivalent to finding the zeros of a polynomial in $f$. This a well-known problem, and can be solved efficiently by, for example, the Newton-Raphson method.

The above analysis leads to a nearly closed form solution for the anomaly detection statistic. One need only insert the solution $f$ to Equation (42) into $L(f)$ (Equation (38)) and compare to a threshold. (If there is no solution with $f$ on the interval $[0,1]$, then $L(f)$ is monotonic on the interval, and the appropriate value of $f$ to use is $\arg \min _{f=0,1}[L(f)]$.) This is equivalent to setting the numerator in Equation (19) equal to a constant and thresholding the background pdf, as described earlier.

The decision surface (Figure 7) now does a better job of enfolding possible mixtures. Every pixel test value $x$ in spectral space corresponds to some effective value of $f$, as determined by the above procedure. This in turn corresponds to some covariance and mean, as defined by Equation (24). The test for a target in Equation (19) is therefore just a spectrally local RX algorithm. The full decision surface is the envelope of local anomaly detectors.

For signature-based detection, the numerator in Equation (19) is not constant. However, calling $L^{\prime}(f)$ the negative logarithm of the numerator, its minimization (maximization of the target pdf) procedure can be trivially derived from the one above for $L(f)$. From Equation (21), it follows that one need only substitute $x$ - $t$ for $x$ in the formulae above, to derive the form of $L^{\prime}(f)$ that is to be minimized over $f$. The analogue of the LMF for this 2-cluster SMM background model is therefore the test:

$$
\underset{f}{\operatorname{Min}}\left[L^{\prime}(f)\right]-\operatorname{Min}_{f}[L(f)]<k
$$

with $L(f)$ given by Equation (38) (after the optimal $f$ is found from the above procedure). Equation (38) also gives the form for $L^{\prime}(f)$, after the substitution $x \rightarrow x$ - $t$, to be minimized over $f$. The decision surface cannot be interpreted as easily as for the above anomaly detector, because the values of $f$ that maximize the numerator and denominator of Equation (19) are generally different. Locally the decision surface arises from a quadratic detector and is not simply the envelope of LMF-type hyperplanes. 


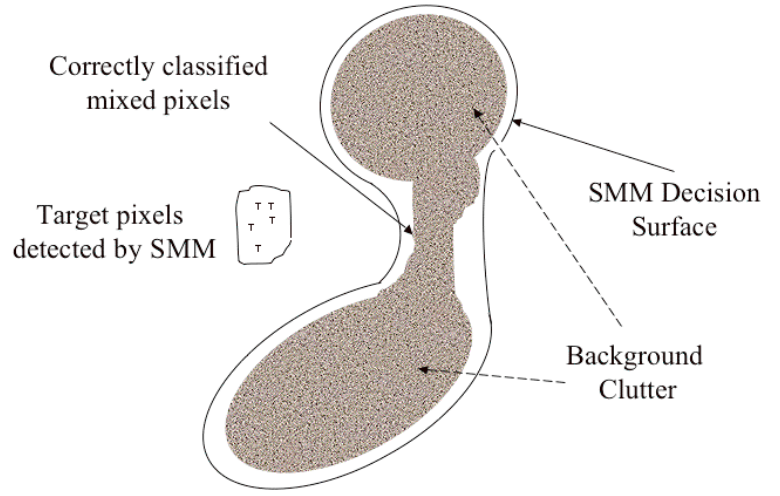

Figure 7. The SMM-based anomaly detector decision surface accurately classifies targets and all background pixels, including mixed ones.

\section{SUMMARY}

Based on concepts borrowed from the Stochastic Mixing Model, a 2-cluster model of hyperspectral background clutter has been used to devise two detection algorithms. The first searches for spectral anomalies, and the second is informed by a mean target signature spectrum. The model is meant to overcome one of the principal remaining problems in autonomous wide-area surveillance with hyperspectral sensing, associated with nonstationary clutter statistics at geographical boundaries.

Mathematical forms for the algorithms have been found that exploit existing signal processing modules in operational systems, as well as in Navy systems designed for rapid prototyping. The only significant new type of computation required is finding the zero of a polynomial function at every test pixel, a straightforward task for the standard Newton-Raphson method. The next step will be a series of tests of these concepts, conducted with experimental hyperspectral imagery.

\section{REFERENCES}

[1] W. Kendall, "The Civil Air Patrol's ARCHER Hyperspectral Detection System," Proc. Specialty Group on Camouflage, Concealment, and Deception, Military Sensing Symposium, 2005.

[2] Alan Stocker, "A. Schaum, Application of stochastic mixing models to hyperspectral detection problems," Proc. of SPIE, Vol. 3071, 22 April, 1997, pp. 47-60.

[3] P. Masson, W. Pieczynski, "SEM algorithm and Unsupervised Statistical Segmentation of Satellite Images," IEEE Trans. on Geoscience and Remote Sensing, vol. 31, no. 3, pp. 618-633, May, 1993.

[4] C. Chang, "Orthogonal subspace projection (osp) revisited: A comprehensive study and analysis," IEEE Trans. on Geoscience and Remote Sensing, vol. 43, no. 3, pp. 502-518, March, 2005.

[5] S. Johnson, "The constrained signal detector," IEEE Trans. on Geoscience and Remote Sensing, vol. 40, no. 6, pp. 1326-1337, June 2002.

[6] L.L. Scharf, Statistical Signal Processing, AddisonWesley, 1991.

[7] M. Farrell, R. Mersereau, On the Impact of Covariance contamination for Adaptive Detection in Hyperspectral Imaging, IEEE Signal Proc. Letters, vol. 12, NO. 9, Sept. 2005.

[8] Alan Stocker, A. Schaum, Application of stochastic mixing models to hyperspectral detection problems, Proc. of SPIE, Vol. 3071, 22 April, 1997, pp. 47-60.

[9] Stein, Material identification and classification in hyperspectral imagery using the normal mixture model, Proc. of SPIE, Vol. 5093, 21 April, 2003, pp. 559-568.

[10] M.T. Eismann and R.C. Hardie, "Improved initialization and convergence of a stochastic spectral unmixing algorithm," Applied Optics, vol. 43, pp. 65966608 (April 2004).

\section{BIOGRAPHY}

Dr. Alan Schaum received a Ph.D. in Theoretical Physics

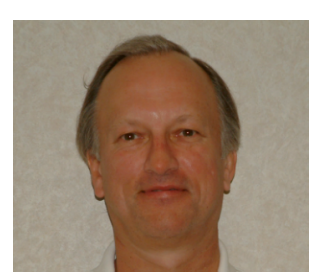
in 1978 from The Johns Hopkins University, where his research focused on relativistic quantum field theory. Until 1983 he applied queuing and information theories to network planning problems at Bell Laboratories in Holmdel, New Jersey.

For the past 22 years at The Naval Research Laboratory in Washington D.C., he has developed signal and image processing methods in support of intelligence operations. His current research emphasizes multivariate detection theory and the modeling of hyperspectral terrestrial background signatures and their dynamics. 